\title{
Correspondence
}

\section{A Proof of the Spherical Homeomorphism Conjecture for Surfaces}

Lowell Abrams, Donniell E. Fishkind, and Carey E. Priebe*

\begin{abstract}
The human cerebral cortex is topologically equivalent to a sphere when it is viewed as closed at the brain stem. Due to noise and/or resolution issues, magnetic resonance imaging may see "handles" that need to be eliminated to reflect the true spherical topology. Shattuck and Leahy [2] present an algorithm to correct such an image. The basis for their correction strategy is a conjecture, which they call the spherical homeomorphism conjecture, stating that the boundary between the foreground region and the background region is topologically spherical if certain associated foreground and background multigraphs are both graph-theoretic trees. In this paper, we prove the conjecture, and its converse, under the assumption that the foreground/background boundary is a surface.
\end{abstract}

Index Terms-Magnetic resonance imaging, segmentation, topology, topological correction.

\section{INTRODUCTION}

The human cerebral cortex is topologically equivalent to a sphere when it is viewed as closed at the brain stem. Due to noise and/or resolution issues, magnetic resonance (MR) imaging may see "handles" that need to be eliminated to reflect the true spherical topology.

Shattuck and Leahy [2] present an algorithm to correct such an image. The basis for their correction strategy is a conjecture, which they call the spherical homeomorphism conjecture, stating that the boundary between the foreground region and the background region is topologically spherical if certain associated foreground and background multigraphs are both graph-theoretic trees.

In this paper, we prove the conjecture, and its converse, under the assumption that the foreground/background boundary is a surface. Note that if the foreground/background boundary is not a surface, then it cannot be topologically spherical.

\section{DEFINITIONS}

For positive integer $N$, we consider the subset of Euclidean 3-space $\mathcal{I}:=\left\{(x, y, z) \in \mathbf{R}^{3}: 0 \leq x, y, z \leq N\right\}$, endowed with the usual Euclidean metric and topology. For all $N^{3}$ triplets of indexes $(i, j, k) \in\{1,2, \ldots, N\}^{3}$ we define the $i, j, k$ th voxel to be the subset of $\mathcal{I}$ given by

$$
\begin{aligned}
v_{i j k}:=\{(x, y, z) & \in \mathcal{I}: \\
& i-1 \leq x \leq i, j-1 \leq y \leq j, k-1 \leq z \leq k\} .
\end{aligned}
$$

The index $i$ is the height of the voxel $v_{i j k}$. The level $L_{i}$ is the union of all voxels of height $i$. The sheet $S_{i, i+1}$ is the intersection $L_{i} \cap L_{i+1}$

Manuscript received May 8, 2002; revised September 11, 2002. The Associate Editor responsible for coordinating the review of this paper and recommending its publication was J. Prince. Asterisk indicates corresponding author.

L. Abrams is with the Department of Mathematics, George Washington University, Washington, DC 20057 USA.

D. E. Fishkind is with the Department of Mathematical Sciences, Johns Hopkins University, Baltimore, MD 21218-2682 USA.

*C. E. Priebe is with the Department of Mathematical Sciences and the Center for Imaging Science, Johns Hopkins University, Baltimore, MD 21218-2682 USA.

Digital Object Identifier 10.1109/TMI.2002.806590

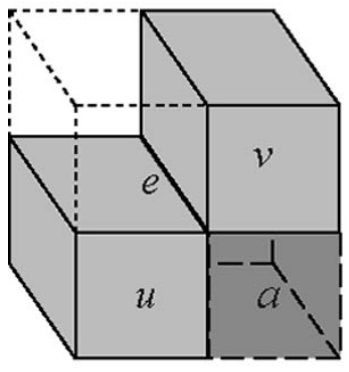

(a)

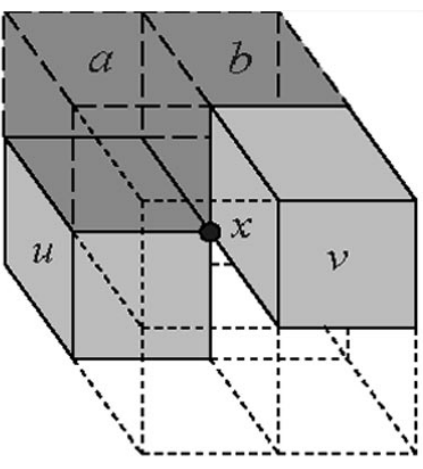

(b)
Fig. 1. Implications of $\partial F G$ being a surface

Each voxel is a cube and, thus, its boundary has six faces, called voxel faces. If the intersection of two distinct voxel faces is a line segment, we call the intersection a voxel edge. If the intersection of two voxel edges is a point, we call the intersection a voxel vertex. For any set $A \subset \mathbf{R}^{3}$, we denote the boundary of $A$ by $\partial A$. If $A \subset S$ for some sheet $S$, we denote the boundary of $A$ in $S$ by $\partial_{S}(A)$. Note that $\partial A$ is two-dimensional and $\partial_{S}(A)$ is one-dimensional.

Each of the voxels in $\mathcal{I}$ is classified as either foreground or background. For this MR brain imaging application, the segmentation process classifies a voxel as foreground if the corresponding location in the brain consists of tissue which is interior to the cerebral cortex; otherwise it classifies the voxel as background. Define $F G$ as the union of all foreground voxels and $B G$ as the union of all background voxels. We make the following two important assumptions: First, we assume that $\partial F G$ is topologically equivalent to an orientable surface, which includes the assumption that $\partial F G$ is connected. For this brain imaging application, the surface $\partial F G$ is understood to be an approximate representation of the cerebral cortex itself. Thus, in principle, it should be topologically spherical, but may be of higher genus due to noise and/or resolution issues. The second assumption is that foreground is surrounded by background, i.e., $\partial \mathcal{I} \cap \partial F G=\emptyset$. In other words, the foreground image is strictly contained in $\mathcal{I}$.

For each $i$, each connected component of $L_{i} \cap F G$ is called a foreground vertex of height $i$. If two foreground vertices of adjacent heights have a nonempty intersection then each connected component of the intersection (which lies in $S_{i, i+1}$ ) is called a foreground edge. (There may be more than one foreground edge between two foreground vertices.) Denote by $V_{f}$ the set of all foreground vertices of all heights, and denote by $E_{f}$ the set of all foreground edges. The foreground graph $G_{f}$ is the (multi)graph $\left(V_{f}, E_{f}\right)$ in which each foreground edge $\epsilon$ in the intersection of foreground vertices $\omega_{1}$ and $\omega_{2}$, say, is considered to be a graph-theoretic edge whose two endpoints are $\omega_{1}, \omega_{2}$. Thus, when we speak of a foreground vertex or a foreground edge we not only think of them as subsets of $\mathcal{I}$, but also as graph-theoretic objects, and the perspective in use at any point of this manuscript will be clear from its context. Analogously, define background vertices, background edges, $V_{b}$ (the set of all background vertices), $E_{b}$ (the set of all background edges), and the background graph $G_{b}=\left(V_{b}, E_{b}\right)$ by substituting $B G$ for $F G$ in the foreground definitions.

The assumption that $\partial F G$ is a surface yields a number of useful implication: 1) If foreground voxels $u$ and $v$ intersect at precisely voxel edge $e$ [see Fig. 1(a)], then at least one voxel $a$ of the other two voxels 
sharing $e$ is foreground. 2) If foreground voxels $u$ and $v$ intersect precisely at voxel vertex $x$ [see Fig. 1(b)], then among the other six voxels sharing $x$ there are foreground voxels $a$ and $b$ such that each of $u \bigcap a$, $a \bigcap b$, and $b \bigcap v$ are voxel faces. (Analogs of these two observations hold, mutatis mutandis, for the background as well.) 3) The boundary of each foreground or background vertex is an orientable surface. 4) For each foreground and background edge $\epsilon$ in, say, sheet $S$ we have that $\partial_{S}(\epsilon)$ consists of (pairwise disjoint) simple closed curves. ${ }^{1}$ 5) For each $i$ and each foreground or background vertex $\omega$ in $L_{i}$ or $L_{i+1}$, the region $\omega \cap S_{i, i+1}$ is connected and its boundary in $S_{i, i+1}$ is a disjoint union of simple closed curves. 6) The graphs $G_{f}$ and $G_{b}$ are both connected.

Formally, the foreground and background graphs in [2] are constructed differently than is done here. There, the foreground graph is modified using a "special connectivity rule," and adjacency for background vertices is defined using the "D18" rule (which considers sharing of a voxel edge an adjacency). However, under the assumption that $\partial F G$ is a surface, the observations in the previous paragraph show that the "special connectivity rule" never applies, and that the D18 rule defines an adjacency exactly when the construction here does. Thus, when $\partial F G$ is a surface, the foreground and background graphs in [2] are exactly $G_{f}$ and $G_{b}$ as constructed here.

For all $i$, let $\left|V_{f_{i}}\right|,\left|V_{b_{i}}\right|,\left|E_{f_{i, i+1}}\right|$, and $\left|E_{b_{i, i+1}}\right|$ denote the number of foreground vertices of height $i$, background vertices of height $i$, foreground edges with endpoints of heights $i$ and $i+1$, and background edges with endpoints of heights $i$ and $i+1$, respectively.

\section{MAIN RESULT}

Shattuck and Leahy [2] conjectured that if both their foreground and background multigraphs are trees, then $\partial F G$ is topologically equivalent to a sphere. Our result regarding their conjecture is the following theorem, which restricts attention to the case in which $\partial F G$ is a surface. ${ }^{2}$

Theorem 1: Suppose that $\partial F G$ is a surface. Then $\partial F G$ is topologically equivalent to a sphere if and only if both $G_{f}$ and $G_{b}$ are trees.

The graph $G_{f}\left(G_{b}\right)$ is a tree if and only if $\left|E_{f}\right|=\left|V_{f}\right|-1\left(\left|E_{b}\right|=\right.$ $\left.\left|V_{b}\right|-1\right)$. By Theorem 1 any extra edges beyond this amount indicates that $\partial F G$ is not topologically spherical. In general, there is not necessarily a way to determine the genus of $\partial F G$ exactly from the number of excess edges, but there is one special case where this can be done:

Theorem 2: Suppose that $\partial F G$ is a surface and that $\forall \omega \in V_{f}$ the genus of $\partial \omega$ is zero. Then the genus of $\partial F G$ is $\left|E_{f}\right|-\left|V_{f}\right|+1$. Thus, $\partial F G$ is topologically equivalent to a sphere if and only if $G_{f}$ is a tree.

Note that the assumption that the genus of $\partial \omega$ is zero $\forall \omega \in V_{f}$ is equivalent to the assumption that $G_{b}$ is precisely a path of length $N$.

For any two-cell embedding of a graph on an orientable surface of genus $g$ with vertex-set $V$, edge-set $E$ and face-set $F$, a classical result of Euler and Poincaré gives the relationship

$$
\chi(S):=|V|-|E|+|F|=2-2 g
$$

(see, e.g., [3, p. 268]). This quantity $\chi(S)$ is called the Euler characteristic of the surface. If $A$ is the union of any collection of voxels

${ }^{1}$ Each voxel vertex $y$ in $S$ intersects (at most) four voxel faces of $S$. Consideration of cases, together with 1) and 2) above, shows that $y$ intersects exactly zero or two voxel edges of $\partial_{S}(\epsilon)$. Thus, viewing voxel vertices and voxel edges as graph theoretic vertices and edges, respectively, we see that $\partial_{S}(\epsilon)$ consists of disjoint cycles.

${ }^{2}$ Notice that in the topological sense $\partial F G$ is obtained from a disjoint union of spheres (i.e., boundaries of voxels) via finitely many direct sums (adjacencies of voxel faces) and point identifications (adjacencies of voxel edges or vertices). Thus, if $\partial F G$ is not a surface, then it is either not connected or, topologically, it arises from a surface via point identifications; in neither case is it topologically equivalent to a sphere. then $\partial A$ has a natural two-cell embedding with the voxel vertices, edges, and faces on the boundary $\partial A$ playing the role of graph-theoretic vertices, edges, and faces, respectively. The Euler characteristic $\chi(\partial A):=|V|-|E|+|F|$, where $V, E$, and $F$ denote the sets of voxel vertices, edges, and faces on $\partial A$, is a well-defined topological invariant even if $A$ is not a surface.

One approach to determining the genus of $\partial F G$ is to determine its Euler characteristic. This may be done on a local basis, by summing $\chi(\partial \omega)$ over all $\omega \in V_{f}$ and then adjusting for the voxel vertices, edges, and faces that were overcounted. An overcount occurs when a voxel face is on two different foreground vertex boundaries, hence is in a foreground edge and is not on $\partial F G$. Such a voxel face needs to be subtracted away twice, once for each of the two times it was (erroneously) counted. Note that if $\epsilon$ is a foreground edge in sheet $S$, then all the voxel edges and vertices that are not in $\partial_{S}(\epsilon)$ were overcounted twice, and the voxel edges and vertices in $\partial_{S}(\epsilon)$ were overcounted once since they are in $\partial F G$ (once) but were counted twice.

If $\epsilon$ is a foreground edge in sheet $S$ with $n_{\epsilon}$ voxel vertices, $e_{\epsilon}$ voxel edges, and $f_{\epsilon}$ voxel faces, the net amount that must be added to the global Euler characteristic count is

$$
\chi^{\prime}(\epsilon):=-2\left(n_{\epsilon}-e_{\epsilon}+f_{\epsilon}\right) .
$$

This is because the components of $\partial_{S}(\epsilon)$ are simple, closed curves and, thus, they have exactly as many voxel edges as voxel vertices; since contributions from edges and vertices have opposite sign in (2), the fact that we subtract them one more time (that is, the second time) has no net effect.

Indeed, if we set $\chi_{i, i+1}^{\prime}:=\sum_{\epsilon \in E_{f_{i, i+1}}} \chi^{\prime}(\epsilon)$, then we have

$$
\begin{aligned}
\chi(\partial F G) & =\sum_{\omega \in V_{f}} \chi(\partial \omega)+\sum_{\epsilon \in E_{f}} \chi^{\prime}(\epsilon) \\
& =\sum_{\omega \in V_{f}} \chi(\partial \omega)+\sum_{i=1}^{N-1} \chi_{i, i+1}^{\prime} .
\end{aligned}
$$

The boundary of each foreground vertex is a surface, and the handles of these foreground-vertex-surfaces are in one-to-one correspondence with the set of all background vertices except for the $N$ background vertices that intersect $\partial I$. Thus, we have

$$
\sum_{\omega \in V_{f}} \chi(\partial \omega)=2\left|V_{f}\right|-2\left|V_{b}\right|+2 N
$$

which gives us the first summand in right-hand side of (3).

To get the second summand in right-hand side of (3), we need two lemmas. Let $T_{i, i+1}$ denote the complement in $S_{i, i+1}$ of the union of foreground edges, and let $t_{i, i+1}$ denote the number of connected regions of $T_{i, i+1}$.

Lemma 3: For all $i, \chi_{i, i+1}^{\prime}=-2-2\left|E_{f_{i, i+1}}\right|+2 t_{i, i+1}$.

Proof of Lemma 3: Consider $S_{i, i+1}$ with its embedded voxel vertices, edges, and faces. Just for this proof, disregard all voxel edges and vertices not contained in a foreground edge, and merge each of the $t_{i, i+1}$ connected components $A$ of $T_{i, i+1}$ to a single face without any embedded voxel vertices, edges, or faces in the interior of $A$. The remaining foreground-edge voxel vertices, edges, and faces and the regions of $T_{i, i+1}$ together constitute a two-cell embedding in $S_{i, i+1}$ of a planar graph $G^{\prime}$. The faces of the embedding are precisely the foreground-edge voxel faces and the connected components of $T_{i, i+1}$, and the number of components of $G^{\prime}$ is exactly $\left|E_{f_{i, i+1}}\right|$. Euler's for- 
mula shows that $\mid\left\{\right.$ vertices of $\left.G^{\prime}\right\}|-|\left\{\right.$ edges of $\left.G^{\prime}\right\}|+|\{$ faces of $\left.G^{\prime}\right\}|=1+| E_{f_{i, i+1}} \mid$. This yields

$$
\begin{aligned}
\chi_{i, i+1}^{\prime} & \equiv \sum_{\epsilon \in E_{f_{i, i+1}}} \chi^{\prime}(\epsilon) \stackrel{(2)}{=}-2 \sum_{\epsilon \in E_{f_{i}, i+1}}\left(n_{\epsilon}-e_{\epsilon}+f_{\epsilon}\right) \\
& =-2\left(t_{i, i+1}+\sum_{\epsilon \in E_{f_{i, i+1}}}\left(n_{\epsilon}-e_{\epsilon}+f_{\epsilon}\right)\right)+2 t_{i, i+1} \\
& =-2\left(1+\left|E_{f_{i, i+1}}\right|\right)+2 t_{i, i+1}
\end{aligned}
$$

which was to be shown.

The following lemma provides a bound for one of the terms in the expression for $\chi_{i, i+1}^{\prime}$ obtained in Lemma 3.

Lemma 4: For all $i, t_{i, i+1} \geq\left|V_{b_{i}}\right|+\left|V_{b_{i+1}}\right|-\left|E_{b_{i, i+1}}\right|$.

Proof of Lemma 4: Let $H$ denote the [bipartite] subgraph of $G_{b}$ induced by $V_{b_{i}} \cup V_{b_{i+1}}$, and let $\pi$ denote the orthogonal projection of $L_{i} \cup L_{i+1}$ on $S_{i, i+1}$. Every path in $H$ between vertices $\omega_{1}, \omega_{2}$ of $H$ is mapped by $\pi$ into $T_{i, i+1}$, and every path in $T_{i, i+1}$ from $\pi\left(\omega_{1}\right)$ to $\pi\left(\omega_{2}\right)$ can be lifted to a path in $H$ from $\omega_{1}$ to $\omega_{2}$. Thus, $H$ has exactly $t_{i, i+1}$ components. For each of these the number of vertices minus the number of edges is at most 1 . Summing over each of the $t_{i, i+1}$ graph-theoretic components of $H$ yields the assertion of the lemma. $\square$

We are now in a position to prove the main results.

Proof of Theorem 1: The characteristic of $\partial F G$ may now be computed and bounded

$$
\begin{aligned}
& \chi(\partial F G) \stackrel{(3)}{=} \sum_{\omega \in V_{f}} \chi(\partial \omega)+\sum_{i=1}^{N-1} \chi_{i, i+1}^{\prime} \\
& \text { (4), }=2\left|V_{f}\right|-2\left|V_{b}\right|+2 N \\
& +\sum_{i=1}^{N-1}\left(-2-2\left|E_{f_{i, i+1}}\right|+2 t_{i, i+1}\right) \\
& \stackrel{\mathrm{L} m 4}{\geq} 2\left|V_{f}\right|-2\left|V_{b}\right|+2+\sum_{i=1}^{N-1}\left(2\left|V_{b_{i}}\right|+2\left|V_{b_{i+1}}\right|\right. \\
& \left.-2\left|E_{f_{i, i+1}}\right|-2\left|E_{b_{i, i+1}}\right|\right) \\
& \left|V_{b_{1}}\right|=\left|V_{b_{N}}\right|=1 \quad 2-2\left[\left(\left|E_{f}\right|-\left|V_{f}\right|+1\right)\right. \\
& \left.+\left(\left|E_{b}\right|-\left|V_{b}\right|+1\right)\right] \text {. }
\end{aligned}
$$

If $G_{f}$ and $G_{b}$ are both trees, then $\left|E_{f}\right|-\left|V_{f}\right|+1=0$ and $\left|E_{b}\right|-$ $\left|V_{b}\right|+1=0$ and, thus, by (8)-(11), we have $\chi(\partial F G) \geq 2$. Since the genus of $\partial F G$ is at least 0 , it follows that $\chi(\partial F G)=2$, which means precisely that $\partial F G$ is topologically equivalent to a sphere.

To prove the converse, suppose without loss of generality that $G_{f}$ contains a cycle $C$. Let $l_{f}$ be a closed curve in $F G$ that "realizes" $C$, i.e., $l_{f}$ lies in the interior of the union of the vertices of $C$, and traverses those vertices in the [unoriented] cyclic order given by $C$. Without loss of generality, we may assume that the intersection of $l_{f}$ with any foreground edge from $C$ consists of exactly one point, and also that each intersection of $l_{f}$ with a sheet is in a foreground edge. Let $S$ be a sheet which intersects at least one edge of $C$. The intersection $S \cap l_{f}$ is a disjoint union of finitely many points $p_{1}, \ldots, p_{n}$ and, because each $p_{i}$ lies in a distinct foreground edge $\epsilon_{i}$, any path from $p_{i}$ to $p_{j}$, $i \neq j$, must pass through $B G$. There is necessarily an $m$ such that the "unbounded" component of the complement of $\epsilon_{m}$ in $S$ contains all other $\epsilon_{i}$ and, thus, there exists a simple closed curve $l_{b}$ in $S \bigcap B G$ such that one region of $S-l_{b}$ contains $p_{m}$, and all other points $p_{i}$ lie in the other region. It is clear that $l_{f}$ and $l_{b}$, as closed curves in $\mathbf{R}^{3}$, are linked and, thus, $l_{f}$ and $l_{b}$ are not contractible in $F G$ and $B G$, respectively. This contradicts the generalized Schoenflies theorem [1], which ensures that both regions of the complement of any topological sphere "nicely" embedded in $\mathbf{R}^{3}$ are simply connected (all loops can be contracted to a point). It follow that $\partial F G$ is not a sphere.

Proof of Theorem 2: The assumption on genus implies that for all $\omega \in V_{f}, \partial \omega$ is a topological sphere and, thus, $\chi(\partial \omega)=2$. If two foreground vertices $\omega_{1}$ and $\omega_{2}$ intersecting in sheet $S$ have exactly one foreground edge $\epsilon$ between them, then $\partial_{S}(\epsilon)$ consists of exactly one simple closed curve that is contractible to a point. Hence, $\partial\left(\omega_{1} \bigcup \omega_{2}\right)$ is also topologically spherical. Thus, we must have $\chi^{\prime}(\epsilon)=-2$ in order to balance $\chi\left(\partial \omega_{1}\right)+\chi\left(\partial \omega_{2}\right)+\chi^{\prime}(\epsilon)=\chi\left(\partial\left(\omega_{1} \bigcup \omega_{2}\right)\right)$. If there are multiple edges, then it follows from the fact that $\chi^{\prime}(\epsilon)$ is calculated locally that each edge $\epsilon \in E_{f}$ has $\chi^{\prime}(\epsilon)=-2$. Thus, by (3), we have

$$
\begin{aligned}
\chi(\partial F G) & =\sum_{\omega \in V_{f}} \chi(\partial \omega)+\sum_{\epsilon \in E_{f}} \chi^{\prime}(\epsilon) \\
& =2\left|V_{f}\right|-2\left|E_{f}\right| \\
& =2-2\left(\left|E_{f}\right|-\left|V_{f}\right|+1\right) .
\end{aligned}
$$

It is clear from (14) and the Euler-Poincaré relationship that the genus of $\partial F G$ is, thus, $\left|E_{f}\right|-\left|V_{f}\right|+1$, and this quantity is zero precisely when $G_{f}$ is a tree, since $G_{f}$ is connected.

Referee comments suggested an alternative, more geometric argument for the proof of Theorem 2: The assumption on genus implies that all $\omega$ in $V_{f}$ are topologically three-balls. Since each foreground edge $\epsilon$ is contractible, gluing the $\omega$ together along the edges $\epsilon$ yields a subspace which deformation-retracts to a complex that is graph-isomorphic to $G_{f}$.

\section{ACKNOWLEDGMENT}

The authors would like to thank the anonymous referees for helpful comments.

\section{REFERENCES}

[1] M. Brown, "A proof of the generalized Schoenflies theorem," Bull. Amer. Math. Soc., vol. 66, pp. 74-76, 1960.

[2] D. W. Shattuck and R. M. Leahy, "Automated graph-based analysis and correction of cortical volume topology," IEEE Trans. Med. Imag., vol. 20, pp. 1167-1177, Nov. 2001.

[3] D. B. West, Introduction to Graph Theory, 2nd ed. Upper Saddle River, NJ: Prentice-Hall, 2001. 